\section{Organic decline}

\author{
M.F. Lappert
}

C, Molecule Chemistry: An International Journal. Editor-in-chief Igor B. Tkatchenko. Harwood. 6/yr. UK $£ 208$ (corporate), £166 (institutional), £83.20 (individual); North America $\$ 250$ (corporate), $\$ 200$ (institutional), $\$ 100$ (individual).

IN THE late 1970 s, when there was concern about the depletion of the world's petroleum reserves, a significant amount of research was centred on alternative sources of organic chemicals. A major part of this endeavour was the conversion of synthesis gas, a mixture of carbon monoxide and hydrogen, into methanol, and thence into organic chemicals containing $\mathrm{C}-\mathrm{C}$ bonds.

The scope of $C_{1}$ Molecule Chemistry is restricted to this area of research which, in the past three or four years, has become less urgent. Many large companies are no longer particularly active in the field, and there is no reason to believe that any great increase in research effort is to be expected in the near future. It is perhaps for this reason that although the first number of Vol. 1 of the journal appeared in April 1984 , No. 5 was published only as recently as January 1986.

The editor-in-chief, the regional editor and the editorial board members are all well-known practitioners of organometallic and/or catalytic chemistry. So far, in the five numbers available for review, some 30 papers have appeared and many of them have one of the board members among the authors. Hence, the overall quality of the contributions is good, but they could well have been published in better established journals and thus have reached a much larger readership. Most of the papers are long, with substantial experimental sections, but there have also been five preliminary communications and a few book reviews.

The journal is of small format $(15 \times 23$ $\mathrm{cm}$ ) and the print is attractively produced, with good quality illustrations. The publication times, however, are quite variable; the worst example appears to be that of a paper which appeared in the fifth issue, but which was received in September 1984. Thus far, Vol. 1 amounts to 422 pages, which means the subscription price is on the expensive side.

While I would acknowledge that some useful papers have appeared in $C_{1}$ Molecule Chemistry, it is not clear that the journal fills an important gap in the literature. Moreover, the extent of specialization is such that it is unlikely that it will have a good chance of surviving.

M.F. Lappert is a Professor in the School of Chemistry and Molecular Sciences, University of Sussex, Brighton BN1 9QJ, UK.

\title{
Under analysis in Japan
}

\section{John M. Ottaway}

Analytical Sciences: The International Journal of the Japan Society for Analytical Chemistry. Editor S. Fujiwara. Katakura Libri, Inc., 36-9, Hongo 3-chome, Bunkyo-ku, Tokyo 113, Japan. 6/yr. \$120 (institutional); $\$ 60$ (individual).

WHILST the appearance of another general journal in the field of analytical science will no doubt be disappointing to many, this one may well serve a very useful purpose if it encourages Japanese authors to make more of their work available in the English language. Analytical Sciences is the new bimonthly journal of the Japan Society for Analytical Chemistry, and to date almost all communications have originated from Japanese authors, and all are in English.

Three categories of article are included, original papers, letters to the editor and "instrumental achievements". The quality of the original papers is, as one would expect, very high indeed, and the subjects covered are very wide with an emphasis on techniques undergoing intense development such as atomic spectrometry, liquid and gas chromatography, electroanalytical methods and flow injection analysis. Average publication times appear to be of the order of five months, with several papers in the April 1986 issue published only two months after submission. Original papers are expected to be six pages or less in length including figures and tables (maximum double-spaced manuscript typescript 18 pages inclusive), whilst the other types of article are restricted to less than two pages.

In the publicity material it is stated that letters to the editor are concerned with opinions of authors working at the forefront of analytical chemistry, and will provide a brief overview of current and future trends, but published articles in this section appear to be mainly shorter papers describing original work. The section called instrumental achievements also

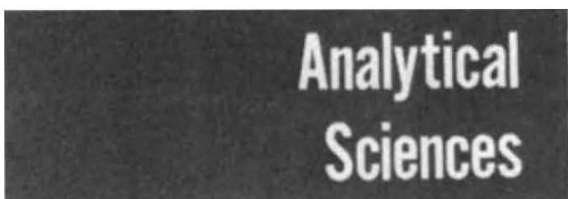

appears to consist mainly of shorter original contributions, but with an emphasis on applications of modern instrumentation and with a preponderance of articles from industrial sources. This seems a particularly worthwhile idea, as too often analytical scientists in industry seem reluctant to publish the excellent work carried out in their laboratories because of the need to complete a development project in sufficient detail for a full or in this case original paper.

The journal also includes a calendar of forthcoming meetings, which contains a listing of many Japanese meetings as well as the main international conferences. Analytical colleagues outside Japan might find this more useful if more details were included about the Japanese events.

The quality of the English and standard of production throughout the journal are excellent, with beautiful coloured photographs in several issues With the rapid development of analytical chemistry and instrumentation in Japan, it is perhaps timely that a vehicle allowing wider dissemination and appreciation of the numerous ideas and new technologies should appear. The journal will be essential reading on a world-wide basis, and international authors may find the short publication times, if they can be maintained, very attractive. As yet another new journal to be incorporated into ever-tightening library budgets, it may be difficult to sell, but there is no doubt that the concept and information will be widely appreciated by the analytical community.

John M. Ottaway is a Professor in the Department of Pure and Applied Chemistry, University of Strathclyde, Cathedral Street, Glasgow G1 IXL, UK.

\section{Surface covering}

\section{Douglas H. Everett}

Langmuir: The ACS Journal of Surfaces and Colloids. Editor Arthur W. Adamson. American Chemical Society. 6/yr. US $\$ 329$ (institutional), \$55 (individual); UK $\$ 342$ (institutional), $\$ 68$ (individual).

Langmuir was launched in January 1985 , with the intention of providing a medium for the publication of both theoretical and experimental work in a wide range of areas of surface and colloid chemistry: solid surfaces in ultra-high vacuum, including surface spectroscopy and structural studies; chemistry and electrochemistry of surfaces; heterogeneous catalysis; all aspects of liquid-liquid and liquidvapour interfaces; and the great domain of disperse systems. The emphasis, it is stated, is to be upon fundamental science rather than on studies of practical importance. This breadth of coverage is reflected in the name chosen for the journal: few scientists have contributed so widely, nor initiated so many new lines of research in surface and colloid chemistry as did Irving Langmuir.

The new journal has two stated missions: first, to exert a unifying influence to 